\title{
Random times at which insiders can have free lunches
}

\author{
Peter Imkeller \\ Institut für Mathematik \\ Humboldt-Universität zu Berlin \\ Unter den Linden 6 \\ 10099 Berlin \\ Germany
}

August 22, 2001

\begin{abstract}
We consider models of time continuous financial markets with a regular trader and an insider who are able to invest into one risky asset. The insider's additional knowledge consists in his ability to stop a random time which is inaccessible to the regular trader, such as the last passage of a certain level before maturity by some stock price process, or the time at which the stock price reaches its maximum during the trading interval. We show that under very mild assumptions on the coefficients of the diffusion process describing these price processes the information drift caused by the additional knowledge of the insider cannot be eliminated by an equivalent change of probability measure. As a consequence, all our models allow the insider to have free lunches with vanishing risk, or even to exercise arbitrage.
\end{abstract}

1991 AMS subject classifications: primary $60 \mathrm{~J}$ 60, 90 A 09; secondary $60 \mathrm{H}$ 30, $60 \mathrm{G} 48$.

Key words and phrases: financial markets, insider, progressive enlargement of filtrations, arbitrage, free lunch, honest time, Brownian motion, diffusion.

\section{Introduction}

In recent years, the study of mathematical models of financial security markets with agents on different information levels has been intensifying. Many papers deal with two species of agents: a regular trader whose information horizon coincides with the natural evolution of the underlying stock price processes, and an insider who possesses some additional information from the beginning of the trading interval, to be revealed to the regular trader continuously until the end of this interval. Work begun by Karatzas and 
Pikovski [29] and Elliott et al [17] deals with the question how the insider's extra utility arising from his additional knowledge can be described. It led to an identification of the optimal logarithmic utility of the insider with the entropy of his additional information in Amendinger [1] and Amendinger, Imkeller and Schweizer [2], and to a more general quantification of the value of initial information in Amendinger, Becherer and Schweizer [3] . In Grorud and Pontier [19] and [20] the insider's optimal portfolios are studied, and on this basis the question is discussed how to detect an insider acting according to these policies in a simple statistical test. Questions of completeness of the insider market model and risk neutral probabilities are discussed in Grorud, Pontier [21], and some simple arbitrage opportunities for the insider are identified. In Imkeller, Pontier and Weisz [24] Malliavin calculus techniques for measure valued processes were used to treat questions of the existence of equivalent martingale measures for the insider's information horizon, and of the existence of arbitrage strategies in a rather general framework.

The common method employed in all these studies is the powerful technique of grossissement de filtrations. It was developed in a series of deep works, e.g. Yor [38], [39], [40], [41], Jeulin [28], Jacod [26]. More precisely, all the above quoted works deal with the simplest version of enlargement of filtrations, the initial enlargement. In this framework, the underlying natural filtration is enlarged by the information about some given random variable for the whole time horizon. In the context of insider trading, this type of enlargement is able to model situations in which the insider gets some fixed additional information at the beginning of the trading interval.

In contrast to this, the extra information the insider is allowed to have in this paper comes from a continuous flow of additional knowledge about a random time the regular trader is unable to stop. We consider random times such as the time at which a Brownian motion or a regular one dimensional diffusion representing possible stock price processes take their maxima, or the last passage time of Brownian motion or a regular one dimensional diffusion through a certain level modelling for example a last level crossing before maturity. Of course, the regular trader will be unable to take action at such a time. We suppose that the insider's information horizon at any time is just big enough so that he is able to stop this random time, and eventually incorporate this knowledge into portfolio decisions. Mathematically, this just means that his filtration is given by the progressive enlargement of the regular trader's filtration. This type of enlargement has been well understood in the pioneering papers on the grossissement techniques. See Yor [39], [40], [41], [42], Jeulin [28]. Our main aim is to find out whether the additional knowledge of one of the random times described above may lead to free lunches or arbitrage for the insider, or whether the information drift favouring the decisions of the insider still allows an equivalent change of probability measure which eliminates this drift from the insider's perspective. In fact, we show that in all the cases considered, excursion theory for Brownian motion can be applied to prove that no equivalent change of probability measure is possible. The principal common reason is the appearance of a three dimensional Bessel process in the information drift. The influence of these processes had already led to similar conclusions about the existence of arbitrage in quite different situations: see Delbaen, Schachermayer [13], or Imkeller, Pontier and Weisz [24]. The more subtle ones of the arbitrage strategies for the insider 
exhibited rely upon the observation that the Brownian motion just after one of the honest times considered behaves like a three dimensional Bessel process, and therefore has a drift which is not noticeable for the regular trader.

The paper is organized as follows. In section 1 the model is explained, some basic facts about progressive enlargements of filtrations are recalled, and a sufficient condition derived under which no equivalent change of measure can make the information drift due to the insider's additional knowledge disappear. In section 2 we consider the enlargement which enables the insider to stop a last level crossing by a Brownian motion. A time reversal argument shows that the information drift contains a component comparable to a 3-dimensional Bessel process which creates arbitrage possibilities. Section 3 is devoted to the study of the random time at which a Brownian $W$ motion reaches its maximum. The well known equality in law of $\sup _{s \leq t} W_{s}-W_{t}, t \geq 0$, with a Brownian motion reflected at 0 enables us to identify the law of the time of the maximum with the law of the (honest) time at which the reflected Brownian motion has its last passage through 0 , and thus to use the results of the preceding section. In section 4 the insider is able to stop the last passage of a level by a regular recurrent diffusion. A time change argument allows to invoke the results of section 2 again. Another time change argument is used in section 5 to show that the insider has free lunches if he can stop the time of the maximum of a regular recurrent diffusion whose volatility is bounded below by a positive constant. The time of the maximum corresponds to some random time for a Brownian motion which can be estimated below by the Brownian motion's time of maximum. This may be treated by the results of section 3 .

\section{The model and some basic facts}

Our basic probability space is the 1-dimensional canonical Wiener space $(\Omega, \mathcal{F}, P)$, equipped with the canonical Wiener process $W=\left(W_{t}\right)_{t \geq 0}$. More precisely, $\Omega=$ $C\left(\mathbf{R}_{+} ; \mathbf{R}\right)$ is the set of continuous functions on $\mathbf{R}_{+}$starting at $0, \mathcal{F}$ the $\sigma$-algebra of Borel sets with respect to uniform convergence on compact subsets of $\mathbf{R}_{+}, P$ Wiener measure and $W$ the coordinate process. The natural filtration $\left(\mathcal{F}_{t}\right)_{t \geq 0}$ of $W$ is assumed to be completed by the sets of $P$-measure 0 .

The financial markets considered will have time horizon 1, be based on Brownian motion, and described by simple one dimensional models. A financial market $(b, \sigma)$ consists of a progressively measurable mean rate of return process $b$ which satisfies $\int_{0}^{1}\left|b_{t}\right| d t<\infty P$-a.s. and of a progressively measurable volatility process $\sigma$ satisfying $\int_{0}^{1} \sigma_{t}^{2} d t<\infty P$-a.s. They determine a (stock) price process given by

$$
\frac{d S_{t}}{S_{t}}=\int_{0}^{t} b_{u} d u+\int_{0}^{t} \sigma_{u} d W_{u} .
$$

For convenience, we let $S_{0}=1$. A progressively measurable process $\pi$ is called a portfolio process if

$$
\int_{0}^{1}\left|\pi_{t} b_{t}\right| d t<\infty \quad \text { P-a.s. }
$$


and

$$
\int_{0}^{1}\left|\pi_{t} \sigma_{t}\right|^{2} d t<\infty \quad \text { P-a.s. }
$$

The excess yield process $R$ and gains process $G$ are given by the formulas

$$
d R_{t}=\frac{d S_{t}}{S_{t}}
$$

and

$$
G_{t}=\int_{0}^{t} \pi_{u} d R_{u}
$$

$0 \leq t \leq 1$. The portfolio process is said to be tame if there is some constant $c \in \mathbf{R}$ such that $G_{t} \geq c$ for all $0 \leq t \leq 1$. Let

$$
K_{0}=\left\{G_{1}=\int_{0}^{1} \pi_{s} d R_{s}: \pi \text { is tame }\right\}
$$

and let $C_{0}$ denote the cone of functions dominated by elements of $K_{0}$, i.e. $C_{0}=K_{0}-L_{+}^{0}$. Set $C=C_{0} \cap L^{\infty}$. The semimartingale $R$ is said to satisfy the condition of no arbitrage (NA) if $C \cap L_{+}^{\infty}=\{0\}$, the condition of no free lunch with vanishing risk (NFLVR) if for the closure $\bar{C}$ of $C$ in $L^{\infty}$ we have $\bar{C} \cap L_{+}^{\infty}=\{0\}$.

We shall assume that there are two agents, acting on the basis of asymmetric information levels. The regular agent's information horizon is given by the filtration $\mathcal{F}=\left(\mathcal{F}_{t}\right)_{t \in[0,1]}$ of the underlying Wiener process restricted to the trading interval $[0,1]$. The insider possesses some additional knowledge which will consist in his ability to stop a random time $L$ which is not accessible to the regular agent. His information horizon will therefore be described by a filtration $\mathcal{G}=\left(\mathcal{G}_{t}\right)_{t \in[0,1]}$ which is larger than the regular agent's. In the terminology of the theory of grossissement de filtrations it is a progressive enlargement which just makes the random time a stopping time to the insider.

We now recall some basic facts about progressive enlargements of filtrations, following Jeulin [28] or Yor [42]. Let $L$ be a random time, i. e. a random variable with values in $[0,1]$. Then the progressive enlargement of $\left(\mathcal{F}_{t}\right)_{t \in[0,1]}$ with respect to $L$ is defined as follows. For $t \in[0,1]$, let

$$
\mathcal{G}_{t}=\left[\mathcal{F}_{t} \vee \sigma(L \wedge t)\right]_{+}
$$

and denote the enlarged filtration by $\mathcal{G}=\left(\mathcal{G}_{t}\right)_{t \in[0,1]}$. $\mathcal{G}$ is the smallest filtration (satisfying the usual conditions) for which $L$ is a stopping time. The computation of the compensator of the Wiener process with respect to the enlarged filtration is of central importance to what follows. We shall sketch it in the following remarks. The right continuous version of the supermartingale $P\left(L>t \mid \mathcal{F}_{t}\right), t \in[0,1]$, will be denoted by $Z^{L}$. Let $M^{L}$ be the martingale in its Doob-Meyer decomposition. It is then well known that the process

$$
\tilde{W}_{t}=W_{L \wedge t}-\int_{0}^{t} 1_{[0, L]}(s) \frac{\frac{d}{d t}\left\langle M^{L}, W\right\rangle_{s}}{Z_{s-}^{L}} d s, \quad t \in[0,1],
$$

is a $\mathcal{G}$-Wiener process before $L$. We trivially have $\tilde{W}_{t}=\tilde{W}_{L \wedge t}, t \in[0,1]$. 
For a special class of random times we can say more. A random time $L$ is called honest time if $L$ is the end of a previsible set, i.e. there is a previsible set $\Gamma \subset[0,1] \times \Omega$ such that

$$
L(\omega)=\sup \{t:(t, \omega) \in \Gamma\},
$$

where $\sup \emptyset=0$. If $L$ is an honest time, the above decomposition formula extends in a simple way beyond $L$. In fact, the process

$$
\tilde{W}_{t}=W_{t}-\int_{0}^{t} 1_{[0, L]}(s) \frac{\frac{d}{d t}\left\langle M^{L}, W\right\rangle_{s}}{Z_{s-}^{L}} d s+\int_{0}^{t} 1_{] L, 1]}(s) \frac{\frac{d}{d t}\left\langle M^{L}, W\right\rangle_{s}}{1-Z_{s-}^{L}} d s, \quad t \in[0,1],
$$

is a $\mathcal{G}$-Wiener process. Let us set

$$
\alpha_{t}=1_{[0, L]}(t) \frac{\frac{d}{d t}\left\langle M^{L}, W\right\rangle_{t}}{Z_{t-}^{L}}-1_{] L, 1]}(t) \frac{\frac{d}{d t}\left\langle M^{L}, W\right\rangle_{t}}{1-Z_{t-}^{L}}, \quad t \in[0,1] .
$$

To find answers to the question, whether the insider can have a free lunch or even exercise arbitrage, we have to take his point of view. Formally, this way we obtain a new financial market $(\tilde{b}, \tilde{\sigma})$ with $\tilde{b}_{t}=b_{t}+\sigma_{t} \alpha_{t}, \tilde{\sigma}_{t}=\sigma_{t}, t \in[0,1]$, with respect to the $\mathcal{G}$-Brownian motion $\tilde{W}$. In the following statements we refer to the point of view of the insider, i. e. we argue for the $\mathcal{G}$-Brownian motion $\tilde{W}$, and the financial market $(\tilde{b}, \tilde{\sigma})$. Formally, the $(N F L V R)$ condition in this market is given by the statement of the fundamental asset pricing Theorem by Delbaen, Schachermayer [12]. It is related to the question whether there exists a measure $Q$ equivalent with $P$ such that under $Q$, the excess yield process $R$ is a martingale. Since we have

$$
R=\int_{0}^{\cdot} \sigma_{t} d W_{t}+\int_{0}^{\cdot} b_{t} d t=\int_{0}^{\cdot} \sigma_{t} d \tilde{W}_{t}+\int_{0}^{\cdot} \tilde{b}_{t} d t
$$

$R$ satisfies $(N F L V R)$ if and only if there exists an equivalent probability measure $Q$ such that with $\theta_{t}=\frac{\tilde{b}_{t}}{\sigma_{t}}=\frac{b_{t}}{\sigma_{t}}+\alpha_{t}$ we have

$$
\left.\frac{d Q}{d P}\right|_{\mathcal{G}_{t}}=\exp \left(-\int_{0}^{t} \theta_{s} d \tilde{W}_{s}-\frac{1}{2} \int_{0}^{t} \theta_{s}^{2} d s\right), \quad t \in[0,1]
$$

From this we immediately obtain that the insider will have free lunch opportunities if we can show that on a set of positive $P$-measure, we have

$$
\int_{0}^{1} \theta_{t}^{2} d t=\infty
$$

Namely, this condition implies that $\frac{d Q}{d P}=0$ on a set of positive $P$-measure (see for example Delbaen, Schachermayer [11], or Revuz, Yor [36]). In [24] we saw that in a particular case, (3) is a consequence of a similar non-integrability property of the pseudodrift $\alpha$. We next show that this relationship is, in fact, much more general.

Proposition 1.1 Let $t \in] 0,1]$. Suppose that

$$
\int_{0}^{t} \alpha_{s}^{2} d s=\infty, \quad \text { on a set of positive measure. }
$$

Then we also have

$$
\int_{0}^{t} \theta_{s}^{2} d s=\infty, \quad \text { on a set of positive measure. }
$$


Proof:

Write $c_{t}=\frac{b_{t}}{\sigma_{t}}$ and suppose for simplicity $t=1$. Let us assume that

$$
\int_{0}^{1} \theta_{t}^{2} d t<\infty
$$

$P$-a.s.. Note that $c$ is $\mathcal{F}$-adapted. Hence, by conditioning on $\mathcal{F}_{t}$ we get for $t \in[0,1]$

$$
\left|c_{t}\right| \leq E\left(\left|\theta_{t}\right| \mid \mathcal{F}_{t}\right)+E\left(\left|\alpha_{t}\right| \mid \mathcal{F}_{t}\right)
$$

If we can show

$$
\int_{0}^{1}\left(E\left(\left|\alpha_{t}\right| \mid \mathcal{F}_{t}\right)\right)^{2} d t<\infty
$$

(5) and Jensen's inequality imply $\int_{0}^{1} c_{t}^{2} d t<\infty$, and therefore the contradiction

$$
\int_{0}^{1} \alpha_{t}^{2} d t \leq 4\left[\int_{0}^{1} c_{t}^{2} d t+\int_{0}^{1} \theta_{t}^{2} d t\right]<\infty \quad P-\text { a.s.. }
$$

Now note that by the very definition of $Z^{L}$ we have for $t \in[0,1]$

$$
E\left(\left|\alpha_{t}\right| \mid \mathcal{F}_{t}\right)=\left|\frac{d}{d t}\left\langle M^{L}, W\right\rangle_{t}\right|
$$

so that the inequality of Kunita-Watanabe yields the estimate

$$
\int_{0}^{1}\left(E\left(\left|\alpha_{t}\right| \mid \mathcal{F}_{t}\right)\right)^{2} d t \leq\left\langle M^{L}\right\rangle_{1}
$$

But the boundedness of $Z^{L}$ clearly justifies that $\left\langle M^{L}\right\rangle_{1}$ is finite $P$-a.s.. This proves (6) and thus the proof is complete.

\section{The last level crossing before maturity by Brow- nian motion}

We shall now consider several particular cases of honest times. The questions we address concern possibilities of free lunches or even arbitrage for insiders who are able to stop the considered time. We start with the last level crossing by the Brownian motion for some fixed level, before time 1 (maturity). We shall see that the model allows for free lunches and arbitrage for quite general price processes. Fix $a \in \mathbf{R}$, and let

$$
L=\sup \left\{0 \leq t \leq 1: W_{t}=a\right\} .
$$

It is obvious that $L$ is an honest time, the end of the (previsible) time set the Wiener process spends on the level $a$. We shall now calculate $\alpha$, or rather give a short argument employing time reversal to prove the well known formula, given for example in Yor [42].

Proposition 2.1 Let $F_{t}$ be the distribution function of the law of $\left|W_{t}\right|, t \in[0,1]$. Then for $t \in[0,1]$ we have

$$
Z_{t}^{L}=1-F_{1-t}\left(\left|W_{t}-a\right|\right)
$$




\section{Proof:}

By a simple spatial shift with $a$, we may assume $a=0$. Fix $0 \leq t \leq 1$, and define

$$
B_{t}=W_{1}-W_{1-t}, \quad \tilde{L}_{t}=\sup _{0 \leq s \leq t} B_{s}
$$

We have the following equation

$$
\begin{aligned}
\{L \leq t\} \cap\left\{W_{1}>0\right\} & =\left\{\tilde{L}_{1-t}<B_{1}\right\} \\
& =\left\{\tilde{L}_{1-t}<W_{1}\right\} \\
& =\left\{\tilde{L}_{1-t}-\left(W_{1}-W_{t}\right)<W_{t}\right\} \\
& =\left\{\tilde{L}_{1-t}-B_{1-t}<W_{t}\right\} .
\end{aligned}
$$

Next observe that the processes $\tilde{L}-B$ and $|B|$ have the same law (see Revuz, Yor [36], p. 223). Since, moreover, $\left|B_{1-t}\right|$ is independent of $\mathcal{F}_{t}$, we obtain

$$
P\left(\left\{L \leq t, W_{1}>0\right\} \mid \mathcal{F}_{t}\right)=P\left(\left|B_{1-t}\right|<W_{t} \mid \mathcal{F}_{t}\right)=F_{1-t}\left(W_{t}^{+}\right) .
$$

(7) and a similar argument for the set $\{L \leq t\} \cap\left\{W_{1}<0\right\}$ finally yield the desired equation.

Given the description of Proposition 2.1 it is easy to obtain an explicit formula for $\alpha$.

Proposition 2.2 For $t \in[0,1]$, let $p_{t}$ be the density of the law of $\left|W_{t}\right|, F_{t}$ its distribution function. Then we have for $t \in[0,1]$

$$
\alpha_{t}=-1_{[0, L]}(t) \frac{p_{1-t}\left(\left|W_{t}-a\right|\right)}{1-F_{1-t}\left(\left|W_{t}-a\right|\right)} \operatorname{sgn}\left(W_{t}-a\right)-1_{] L, 1]}(t) \frac{p_{1-t}\left(\left|W_{t}-a\right|\right)}{F_{1-t}\left(\left|W_{t}-a\right|\right)} \operatorname{sgn}\left(W_{t}-a\right) .
$$

\section{Proof:}

The formula follows immediately from the preceding Proposition, and Itô's and Tanaka's formula

$$
\left|W_{t}-a\right|-|a|=\int_{0}^{t} \operatorname{sgn}\left(W_{s}-a\right) d W_{s}+L_{t}^{a}, \quad t \in[0,1],
$$

with the local time $L_{t}$ at level $a$.

Possibilities of arbitrage and free lunch according to the fundamental asset pricing theorem will, just as in Imkeller, Pontier, Weisz [24], be related with integrability properties of the information drift process $\alpha$. Since it is well known that for honest times the semimartingale property is conserved in passing to the enlarged filtration, we do not have to care about $L^{1}$-integrability of $\alpha$. Let us next show that the process is not square integrable on a set of positive measure.

Proposition 2.3 For any $t \in] 0,1]$, we have

$$
\int_{0}^{t} \alpha_{s}^{2} d s=\infty
$$

on a set of positive measure. 


\section{Proof:}

Fix $0 \leq t \leq 1$. Assume without loss of generality that $a=0$. By Proposition 2.2, the following estimate holds

$$
\left|\alpha_{s}\right| \geq 1_{] L, 1]}(s) \frac{p_{1-s}\left(\left|W_{s}\right|\right)}{F_{1-s}\left(\left|W_{s}\right|\right)}=1_{] L, 1]}(s) \frac{\partial}{\partial x} \ln F_{1-s}\left(\left|W_{s}\right|\right), \quad s \in[0,1] .
$$

By Williams' path decomposition (see Revuz, Yor [36], (4.9) Theorem), we can deduce from (8) a critical lower bound for $\alpha$, at least for times immediately after $L$. For this purpose, let

$$
A=\left\{L \leq \frac{1}{2} t, T_{1}<1\right\}
$$

where $T_{1}=\inf \left\{s \geq L, W_{s}=1\right\}$. Clearly $A$ has positive probability. The process

$$
\rho_{s}=W_{s+L}, \quad 0 \leq s \leq T_{1}-L
$$

is a piece of a $B E S(3)$. Hence on the set $A$

$$
\int_{0}^{t} \alpha_{s}^{2} d s \geq \int_{0}^{\left(T_{1}-L\right) \wedge(t-L)}\left(\frac{p_{1-s}\left(\rho_{s}\right)}{F_{1-s}\left(\rho_{s}\right)}\right)^{2} d s .
$$

But the quotient $\frac{p_{1-s}\left(\rho_{s}\right)}{F_{1-s}\left(\rho_{s}\right)}$ is pathwise asymptotically equivalent with $\frac{1}{\rho_{s}}$ as $s \downarrow L$. And it is well known that $\int_{0}^{u} \frac{1}{\rho_{s}^{2}} d s=\infty P$-a.s. for all $\left.\left.u \in\right] 0,1\right]$. See for example Revuz, Yor [36], Chapter VI, (3.19) exercise. Therefore (9) implies the desired equation.

To show that the insider being able to stop the last passage through $a$ before maturity can have free lunches with no risk, we just have to combine the preceding result with Proposition 1.1.

Theorem 2.1 Let $a \in \mathbf{R}$. Suppose the insider's filtration $\mathcal{G}$ is the progressive enlargement of $\mathcal{F}$ with $L$, the last passage through a before time 1 . Then in the insider's model $R$ does not satisfy the condition (NFLVR).

\section{Proof:}

Combine the Propositions 2.3 and 1.1.

We can go a little further and show that there are even arbitrage strategies, at least for a large class of price processes. Intuitively, according to the strategy exhibited in the following example, the insider makes use of a very subtle effect. While the regular trader cannot stop the random time $L$, and therefore will perceive the price process as a Wiener process, the insider will see an effective decay of the price after time $L$ for a short while, perceiving it as the negative of a 3-dimensional Bessel process. He is able to take advantage of this observation.

\section{Example 1:}

Suppose that there exists $p>2$ such that $\left(\frac{b}{\sigma}\right)^{-} \in L_{p}([0,1])$. Then arbitrage possibilities exist. 
To show this, denote first by $q$ the conjugate exponent to $p$. Hölder's inequality gives

$$
\int_{L}^{t}\left(\frac{b_{s}}{\sigma_{s}}\right)^{-} d s \leq(t-L)^{\frac{1}{q}}\left(\int_{L}^{t}\left(\left(\frac{b_{s}}{\sigma_{s}}\right)^{-}\right)^{p} d s\right)^{\frac{1}{p}} .
$$

The crucial point for our argument is the following observation. If $A, \rho$ are given as in the proof of Proposition 2.3, an argument as in the proof of Theorem 4.3 of Imkeller, Pontier, Weisz [24] precising the local path behaviour of $B E S(3)$ processes and using (10) shows that, at least on $A$ we have

$$
\lim _{t \downarrow L} \frac{W_{t}}{(t-L)^{\frac{1}{q}}}=\infty \quad \text { for } \quad q<2 .
$$

Hence the $\mathbf{G}$-stopping time

$$
\tau=\inf \left\{t \geq L: \int_{L}^{t}\left(\frac{b_{s}}{\sigma_{s}}\right)^{-} d s+\frac{1}{2}(t-L)>\frac{W_{t}}{2}\right\} \wedge 1
$$

is strictly bigger than $L$ on $A$. Using this stopping time, we may define our tame strategy. Let

$$
T_{t}=\exp \left(-\int_{0}^{t} \frac{b_{s}}{\sigma_{s}} d s-W_{t}-\frac{1}{2} t\right)
$$

and choose

$$
\pi_{t}=1_{[L, \tau]}(t) 1_{\mathbf{R}_{+}}\left(W_{t}\right) \frac{T_{t}}{T_{L} \sigma_{t}}, \quad t \in[0,1] .
$$

Then we obtain for $t \in[0,1]$

$$
\begin{aligned}
G_{t} & =\left[\exp \left(\int_{\tau}^{t \wedge \mu} \frac{b_{s}}{\sigma_{s}} d s+W_{t \wedge \tau}-\frac{1}{2}(t \wedge \tau-L)\right)-1\right] 1_{\mathbf{R}_{+}}\left(W_{\tau}\right) \\
& \geq-1
\end{aligned}
$$

and

$$
\begin{aligned}
G_{1} & \geq\left[\exp \left(\frac{W_{\tau}}{2}\right)-1\right] 1_{\mathbf{R}_{+}}\left(W_{\tau}\right) \\
& \geq 0 \text { and }>0
\end{aligned}
$$

at least on the set $A$ which has positive measure. This shows that $\pi$ is an arbitrage strategy and therefore the proof is complete.

We remark that the conditions of the preceding example are fulfilled in case $\frac{b}{\sigma}$ is bounded below by a constant.

\section{The time at which Brownian motion reaches its maximum}

We next consider the time when the Brownian motion on $[0,1]$ takes its maximum. Again, we shall prove that the model then allows for free lunches or arbitrage for insiders who can stop this time. For $t \in[0,1]$ let

$$
S_{t}=\sup _{0 \leq s \leq t} W_{s}
$$


and

$$
\sigma=\sup \left\{0 \leq t \leq 1: W_{t}=S_{t}\right\} .
$$

Let $\tau$ be the time $W$ reaches its maximum in $[0,1]$. Then it is well known that $\sigma=\tau$ $P$-a.s. Hence $\tau$ is obviously an honest time. Now let

$$
\begin{aligned}
\rho & =\sup \left\{0 \leq t \leq 1:\left|W_{t}\right|=0\right\} \\
& =\sup \left\{0 \leq t \leq 1: W_{t}=0\right\} .
\end{aligned}
$$

Since $\left(S_{t}-W_{t}\right)_{t \geq 0}$ and $\left(\left|W_{t}\right|\right)_{t \geq 0}$ have the same law, we also know that $\tau$ and $\rho$ have identical laws. Denote by

$$
\tilde{L}_{s}=\sup _{0 \leq h \leq s}\left(W_{t+h}-W_{t}\right)
$$

$s \geq 0$, and let $G_{t}$ be the law of $S_{t}, t \geq 0$. Then

$$
\begin{aligned}
P\left(\tau>t \mid \mathcal{F}_{t}\right) & =P\left(\tilde{L}_{1-t}+W_{t}>S_{t} \mid \mathcal{F}_{t}\right) \\
& =P\left(\tilde{L}_{1-t}>S_{t}-W_{t} \mid \mathcal{F}_{t}\right) \\
& =G_{1-t}\left(S_{t}-W_{t}\right) .
\end{aligned}
$$

So we obtain a simple characterization of the density of the compensator in the following proposition.

Proposition 3.1 For $t \in[0,1]$, let $G_{t}$ resp. $q_{t}$ denote the law resp. density function of $S_{t}$. Then for $0 \leq t \leq 1$ we have

$$
\alpha_{t}=-1_{[0, \tau]}(t) \frac{q_{1-t}\left(S_{t}-W_{t}\right)}{1-G_{1-t}\left(S_{t}-W_{t}\right)}-1_{] \tau, 1]}(t) \frac{q_{1-t}\left(S_{t}-W_{t}\right)}{G_{1-t}\left(S_{t}-W_{t}\right)} .
$$

\section{Proof:}

The random variables $S_{t}$ and $\left|W_{t}\right|$ are equal in law. Write $p_{t}$ for the density of $W_{t}$. Then we have for $t \in[0,1], x \in \mathbf{R}$

$$
H_{t}(x)=\int_{-|x|}^{|x|} p_{t}(y) d y=2 \int_{0}^{|x|} p_{t}(y) d y .
$$

Hence we have

$$
H_{t}(x)=G_{t}(|x|), \quad x \in \mathbf{R}, \quad t \in[0,1] .
$$

So the result of Proposition 2.2 may be paraphrased as

$$
\begin{aligned}
\alpha_{t} & =-1_{[0, L]}(t) \frac{2 p_{1-t}\left(W_{t}\right)}{1-H_{1-t}\left(W_{t}\right)}-1_{] L, 1]}(t) \frac{2 p_{1-t}\left(W_{t}\right)}{H_{1-t}\left(W_{t}\right)} \\
& =-1_{[0, L]}(t) \frac{q_{1-t}\left(\left|W_{t}\right|\right)}{1-G_{1-t}\left(\left|W_{t}\right|\right)}-1_{] L, 1]}(t) \frac{q_{1-t}\left(\left|W_{t}\right|\right)}{G_{1-t}\left(\left|W_{t}\right|\right)} .
\end{aligned}
$$

Note that $L$ corresponds to $\rho$. Now it remains to apply the equality of laws of $\left(S_{t}-\right.$ $\left.W_{t}\right)_{t \geq 0}$ and $\left(\left|W_{t}\right|\right)_{t \geq 0}$ to obtain the desired result. 
Proposition 3.2 For any $t \in] 0,1]$, we have

$$
\int_{0}^{t} \alpha_{s}^{2} d s=\infty
$$

on a set of positive measure.

\section{Proof:}

This again is an immediate consequence of Proposition 2.3 and the equality of the laws of $\left(S_{t}-W_{t}\right)_{t \geq 0}$ and $\left(\left|W_{t}\right|\right)_{t \geq 0}$.

So we obtain the main result of this section.

Theorem 3.1 Suppose the insider's filtration $\mathcal{G}$ is the progressive enlargement of $\mathcal{F}$ with $\tau$, the time at which $W$ reaches its maximum. Then in the insider's model $R$ does not satisfy the condition (NFLVR).

\section{Proof:}

This is a combination of Propositions 3.2 and 2.1.

For large classes of price processes it is equally easy to exhibit arbitrage strategies.

\section{Example 2:}

Suppose that there exists $p>2$ such that $\left(\frac{b}{\sigma}\right)^{+} \in L_{p}([0,1])$. Then there is arbitrage. To show this, we proceed as in the previous example. Let $\tau$ again be the random time when $W$ reaches its maximum, $q$ the conjugate exponent to $p$. Hölder's inequality gives

$$
\int_{\tau}^{t}\left(\frac{b_{s}}{\sigma_{s}}\right)^{+} d s \leq(t-\tau)^{\frac{1}{q}}\left(\int_{\tau}^{t}\left(\left(\frac{b_{s}}{\sigma_{s}}\right)^{+}\right)^{p} d s\right)^{\frac{1}{p}}
$$

Our argument relies upon the following observation. Given $\tau$, the process $\left(\rho_{t}=\left(W_{\tau}-\right.\right.$ $\left.W_{\tau+t}: 0 \leq t \leq 1-\tau\right)$ is a Bes(3) process. Another argument as in Example 1 therefore gives

$$
\lim _{t \downarrow 0} \frac{\rho_{t}}{t^{\frac{1}{q}}}=\infty \text { for } \quad q<2 .
$$

(13) and (14) together imply that on a random, small but nontrivial time interval just after $\tau$, we have

$$
\int_{\tau}^{t}\left(\frac{b_{s}}{\sigma_{s}}\right)^{+} d s<W_{\tau}-W_{t}
$$

Hence the $\mathbf{G}$-stopping time

$$
\mu=\inf \left\{t \geq \tau: \int_{\tau}^{t}\left(\frac{b_{s}}{\sigma_{s}}\right)^{+} d s+\frac{1}{2}(t-\tau)>\frac{W_{\tau}-W_{t}}{2}\right\} \wedge 1
$$

is strictly bigger than $\tau$. Using this stopping time, we may define our tame strategy. Let

$$
T_{t}=\exp \left(-\int_{0}^{t} \frac{b_{s}}{\sigma_{s}} d s-W_{t}-\frac{1}{2} t\right)
$$


and choose

$$
\pi_{t}=-1_{[\tau, \mu]}(t) \frac{T_{t}}{T_{\tau} \sigma_{t}}, \quad t \in[0,1] .
$$

Then we obtain for $t \in[0,1]$

$$
\begin{aligned}
G_{t} & =\left[\exp \left(-\int_{\tau}^{t \wedge \mu} \frac{b_{s}}{\sigma_{s}} d s+\left(W_{\tau}-W_{t \wedge \mu}\right)-\frac{1}{2}(t \wedge \mu-\tau)\right)-1\right] \\
& \geq-1
\end{aligned}
$$

and

$$
\begin{aligned}
G_{1} & \geq\left[\exp \left(\frac{W_{\tau}-W_{\mu}}{2}\right)-1\right] \\
& \geq 0 \text { and }>0
\end{aligned}
$$

with positive probability. This completes the proof.

\section{The last zero crossing by a recurrent diffusion}

Let $\left(X_{t}\right)_{t \geq 0}$ be a recurrent regular diffusion on $\mathbf{R}$, given by a stochastic differential equation

$$
d X_{t}=c\left(X_{t}\right) d t+\gamma\left(X_{t}\right) d W_{t}
$$

where $\gamma^{2}>0$ and $\frac{c}{\gamma^{2}}$ is locally integrable on $\mathbf{R}$. Define

$$
L=\sup \left\{0 \leq t \leq 1: X_{t}=0\right\}
$$

the last crossing of zero before time 1. Obviously, we again treat an honest time. We shall derive conditions on the Feller characteristics of $X$ under which the insider has free lunch or arbitrage possibilities. According to Revuz, Yor [36], p. 321, we have for $t \in[0,1]$

$$
Z_{t}^{L}=\phi\left(X_{t}\right)
$$

where

$$
\phi(x)=P_{x}(L>0)=P_{x}\left(\tau_{0}<1\right) .
$$

Here $\tau_{0}$ denotes the first hitting time of 0 by $X$. To estimate $\phi$ for small $x$, we use classical diffusion theory. Itô, Mc Kean [25], p. 215, yields

$$
\phi(x)-\left[(u(x)-u(0)) n_{+}(1)+1\right]=o(u(x)-u(0)) \quad \text { as } \quad x \rightarrow 0,
$$

where

$$
0>n_{+}(l)=\int_{l}^{\infty} n_{+}(d u)>-\infty
$$

and $n_{+}$is the Lévy measure appearing in the formula

$$
E_{0}\left(e^{-\alpha \mathbf{t}_{+}^{-1}(t)}\right)=e^{-t \int_{0}^{\infty}\left(1-e^{-\alpha l}\right) n_{+}(d l)},
$$


with the local functional

$$
\mathbf{t}_{+}^{-1}(t)=\lambda\left(s: X_{s}>0, \tau_{0} \leq s \leq L^{-1}(t)\right),
$$

and the inverse local time $L^{-1}$ at $0 . u$ denotes the scale function of $X$. Let us first give some estimates for the martingale part of $Z^{L}$. For this purpose, recall that under our assumptions $u$ is continuously differentiable, $Y_{t}=u\left(X_{t}\right)-u(0), t \geq 0$, is a martingale (see Karatzas, Shreve [30], p. 340), and denote $\psi(y)=\phi\left(u^{-1}(y+u(0))\right), y \in[u(-\infty)-$ $u(0), u(\infty)-u(0)]$.

Lemma $4.1 \psi$ is a convex function, $\psi^{\prime}$ is right continuous at 0 with limit $n_{+}(1)$, and we have

$$
\begin{aligned}
M_{t}^{L} & =1+\int_{0}^{t} \psi^{\prime}\left(Y_{s}\right) d Y_{s}, \\
\frac{d}{d t}\left\langle M^{L}, W\right\rangle_{t} & =\psi^{\prime}\left(Y_{t}\right) u^{\prime}\left(u^{-1}\left(Y_{t}\right)\right) \gamma\left(u^{-1}\left(Y_{t}\right)\right),
\end{aligned}
$$

$t \geq 0$.

\section{Proof:}

The statement is implicit in the derivation on pp. 124, 215 of Itô, Mc Kean [25].

With the help of Lemma 4.1 we can now give a lower estimate of the information drift coefficient $\alpha$.

Proposition 4.1 For any $t \in[0,1]$ we have

$$
\int_{0}^{t} \alpha_{s}^{2} d s=\infty
$$

on a set of positive measure.

\section{Proof:}

By Lemma 4.1, we may estimate for $s \in[0,1]$

$$
\left|\alpha_{s}\right| \geq 1_{] L, 1]}(s) \frac{-\psi^{\prime}\left(Y_{s}\right) u^{\prime}\left(u^{-1}\left(Y_{s}\right)\right)\left|\gamma\left(u^{-1}\left(Y_{s}\right)\right)\right|}{1-\psi\left(Y_{s}\right)} .
$$

Since $\psi^{\prime}$ is increasing with limit $n_{+}(1)$ at 0 , and by (16), we may continue this estimate by

$$
\int_{0}^{t} \alpha_{s}^{2} d s \geq \int_{L}^{t \vee L} \frac{u^{\prime}\left(u^{-1}\left(Y_{s}\right)\right)^{2} \gamma\left(u^{-1}\left(Y_{s}\right)\right)^{2}}{Y_{s}^{2}} d s, \quad t \in[0,1] .
$$

We proceed by defining the random time change

$$
A_{t}=\int_{0}^{t} u^{\prime}\left(u^{-1}\left(Y_{s}\right)\right)^{2} \gamma\left(u^{-1}\left(Y_{s}\right)\right)^{2} d s, \quad t \in[0,1] .
$$

Since $A=\langle Y\rangle$, the process

$$
W_{t}=Y_{A_{t}^{-1}}, \quad 0 \leq t \leq A_{1},
$$


is a Wiener process. Moreover, by strict monotonicity of $A$, the random time $A_{L}$ is the last zero crossing of the Wiener process $W$ before time $A_{1}$, and we may write

$$
\int_{0}^{t} \alpha_{s}^{2} d s \geq \int_{A_{L}}^{A_{t} \vee A_{L}} \frac{1}{W_{s}^{2}} d s .
$$

We may now continue our argument as in the proof of Proposition 2.3.

Again, the insider may have free lunches without risk, as our main result shows.

Theorem 4.1 Suppose the insider's filtration $\mathcal{G}$ is the progressive enlargement of $\mathcal{F}$ with $L$, the last passage through zero of a regular recurrent diffusion $Y$ satisfying the stochastic differential equation

$$
d Y_{t}=\gamma\left(Y_{t}\right) d W_{t}+c\left(Y_{t}\right) d t
$$

with coefficients $\gamma$ and $c$ such that $\gamma^{2}>0$ and $\frac{c^{2}}{\gamma^{2}}$ is locally integrable on $\mathbf{R}$. Then in the insider's model $R$ does not satisfy the condition (NFLVR).

\section{Proof:}

Combine Propositions 4.1 and 1.1.

\section{The time at which a recurrent diffusion reaches its maximum}

Let as in the preceding section $\left(X_{t}\right)_{t \geq 0}$ be a recurrent regular diffusion on $\mathbf{R}$, given by the stochastic differential equation

$$
d X_{t}=c\left(X_{t}\right) d t+\gamma\left(X_{t}\right) d W_{t}
$$

Assume in addition that the volatility of $X$ is bounded below, i.e. there is a positive constant $\rho$ such that $\gamma^{2} \geq \rho$ and $\frac{c^{2}}{\gamma^{2}}$ (and thus also $\frac{c}{\gamma}$ ) is locally integrable on $\mathbf{R}$. Let

$$
S_{t}=\sup _{0 \leq s \leq t} X_{s}
$$

and

$$
\tau=\sup \left\{t \leq 1: X_{t}=S_{t}\right\}
$$

Then $\tau$ is again $P$-a.s. equal to the time at which $X$ takes its maximum. We shall show that there are free lunch and arbitrage possibilities again. Our argument is based on a time change which takes $X$ into a Brownian motion. Then we will be able to adapt the results of section 3. One has to be rather careful, however. At first glance, one might be tempted to conjecture that $\left(S_{t}-X_{t}\right)_{t \geq 0}$ is equal in law to the reflected diffusion $\left(\left|X_{t}\right|\right)_{t \geq 0}$, to resume the arguments of section 3. But this is true only under additional assumptions (see Revuz, Yor [36]). 
We start by remarking that our assumptions on $c$ and $\gamma$ allow to assume that $c=0$ altogether. This can be seen by an application of a Girsanov change of probability measure. Furthermore, another simple argument shows that if the result is proved for a martingale diffusion $X$, it holds for a nontrivial constant multiple of $X$ as well. This remark allows us to assume that $\gamma^{2} \geq 1$.

Define for $t \geq 0$

$$
A_{t}=\int_{0}^{t} \gamma^{2}\left(X_{s}\right) d s
$$

Then $A$ is continuous and strictly increasing with inverse process $A^{-1}$, and $\gamma^{2} \geq 1$ implies $A_{1} \geq 1$. It is well known that

$$
Y_{t}=X_{A_{t}^{-1}}, \quad t \geq 0
$$

is a Wiener process with respect to the filtration

$$
\mathcal{H}_{t}=\mathcal{F}_{A_{t}^{-1}}, \quad t \geq 0
$$

We consider the time of the maximum of $Y$ corresponding to $\tau$. For this purpose, let

$$
T_{t}=\sup _{0 \leq s \leq t} Y_{s}, \quad t \geq 0
$$

and define

$$
\xi=\sup \left\{s \leq A_{1}: Y_{s}=T_{s}\right\}
$$

Then it is easy to see that

$$
A_{\tau}=\xi
$$

Since $A_{1}$ is not necessarily deterministic, $\xi$ is not necessarily an honest time for the Wiener process $Y$. But since $A_{1} \geq 1$, we can fortunately compare it to the honest time

$$
\xi_{0}=\sup \left\{s \leq 1: Y_{s}=T_{s}\right\}
$$

We trivially have

$$
\xi_{0} \leq \xi
$$

and we know that $\xi_{0}$ and $\xi$ agree on the set of positive measure $\left\{T_{1}>\sup _{1 \leq t \leq A_{1}} Y_{t}\right\}$. We finally need the counterpart of $\xi_{0}$ in the original scale. It is given by the random time

$$
\tau_{0}=\sup \left\{t \leq A_{1}^{-1}: X_{t}=S_{t}\right\}
$$

Recall the supermartingale $Z^{\tau}$, and let correspondingly

$$
Z^{\tau_{0}}=P\left(\tau_{0}>t \mid \mathcal{F}_{t}\right), \quad Z^{\xi}=P\left(\xi>t \mid \mathcal{H}_{t}\right), \quad Z^{\xi_{0}}=P\left(\xi_{0}>t \mid \mathcal{H}_{t}\right), \quad t \geq 0 .
$$

In this notation, we have

$$
Z_{t}^{\tau}=Z_{A_{t}}^{\xi}, \quad t \geq 0 .
$$

We shall now investigate $Z^{\xi}$, roughly by comparing it to $Z^{\xi_{0}}$, which we know better from section 3. Let

$$
Z^{\tau}=M^{\tau}-B^{\tau}
$$


be the Doob-Meyer decomposition of $Z^{\tau}$ with a martingale $M^{\tau}$ and a bounded increasing process $B^{\tau}$. By the predictable representation property w. r. t. the filtration $\left(\mathcal{F}_{t}\right)_{t \geq 0}$, there exists an adapted process

$$
f=\frac{d}{d t}\left\langle M^{\tau}, W\right\rangle
$$

such that we have

$$
M_{t}^{\tau}=\int_{0}^{t} f_{s} d W_{s}=\int_{0}^{t} \frac{f_{s}}{\gamma\left(X_{s}\right)} d X_{s}
$$

The Doob-Meyer decomposition of $Z^{\xi}$ with respect to $\left(\mathcal{H}_{t}\right)_{t \geq 0}$ is then given by

$$
Z^{\xi}=M^{\xi}-B^{\xi}
$$

where

$$
B_{t}^{\xi}=B_{A_{t}^{-1}}^{\tau}, \quad M_{t}^{\xi}=M_{A_{t}^{-1}}^{\tau}=\int_{0}^{A_{t}^{-1}} \frac{f_{s}}{\gamma\left(X_{s}\right)} d X_{s}=\int_{0}^{t} \frac{f_{A_{s}^{-1}}}{\gamma\left(Y_{s}\right)} d Y_{s} .
$$

Moreover, we have

$$
g=f_{A^{-1}}=\frac{d}{d t}\left\langle M^{\xi}, Y\right\rangle .
$$

For the associated supermartingales $Z^{\tau_{0}}$ and $Z^{\xi_{0}}$ we obtain in the same way analogous decompositions with increasing processes $B^{\tau_{0}}, B^{\xi_{0}}$,

$$
f^{0}=\frac{d}{d t}\left\langle M^{\tau_{0}}, W\right\rangle, g^{0}=f_{A^{-1}}^{0}=\frac{d}{d t}\left\langle M^{\xi_{0}}, Y\right\rangle .
$$

Due to convexity of $-\ln$, we know that $\ln \frac{1}{1-Z^{\xi}}, \ln \frac{1}{1-Z^{\xi_{0}}}$ are submartingales with respect to the filtration $\left(\mathcal{H}_{t}\right)_{t \geq 0}$, as well as $\ln \frac{1}{1-Z^{\tau}}, \ln \frac{1}{1-Z^{\tau_{0}}}$ are submartingales with respect to $\left(\mathcal{F}_{t}\right)_{t \geq 0}$. Ito's formula in the filtration $\mathcal{F}$ and in the enlarged filtration $\mathcal{G}$ yields for any $c>0,0 \leq t<1$

$$
\begin{aligned}
& \ln \frac{1}{1-Z_{t}^{\tau}}-\ln \frac{1}{1-Z_{\tau+c}^{\tau}} \\
& \quad=\int_{\tau+c}^{t} \frac{f_{s}}{1-Z_{s}^{\tau}} d W_{s}-\int_{\tau+c}^{t} \frac{f_{s}}{1-Z_{s}^{\tau}} d B_{s}^{\tau}+\frac{1}{2} \int_{\tau+c}^{t}\left(\frac{f_{s}}{1-Z_{s}^{\tau}}\right)^{2} d s \\
& \quad=\int_{\tau+c}^{t} \frac{f_{s}}{1-Z_{s}^{\tau}} d \tilde{W}_{s}-\int_{\tau+c}^{t} \frac{f_{s}}{1-Z_{s}^{\tau}} d B_{s}^{\tau}-\frac{1}{2} \int_{\tau+c}^{t}\left(\frac{f_{s}}{1-Z_{s}^{\tau}}\right)^{2} d s .
\end{aligned}
$$

To transfer this formula into the scale $t=A_{s}$, we remark that on $\left.] \xi, 1\right]$ we may write

$$
\begin{aligned}
d \tilde{W}_{t} & =d W_{t}+\frac{f_{t}}{1-Z_{t}^{\tau}} d t \\
& =\frac{1}{\gamma\left(X_{t}\right)} d X_{t}+\frac{f_{t}}{1-Z_{t}^{\tau}} d t \\
& =\frac{1}{\gamma\left(Y_{s}\right)} d Y_{s}+\frac{g_{s}}{\left(1-Z_{s}^{\xi}\right) \gamma^{2}\left(Y_{s}\right)} d s
\end{aligned}
$$


Hence if we define

$$
\tilde{Y}_{t}=Y_{t}+\int_{0}^{t} 1_{] \xi, 1[}(s) \frac{g_{s}}{\left(1-Z_{s}^{\xi}\right) \gamma\left(Y_{s}\right)} d s
$$

we see that $\tilde{Y}$ is a Wiener process with respect to $\left(\left(\mathcal{H}_{t} \vee \sigma(\xi \wedge t)\right)_{+}\right)_{t \geq 0}$ on $] \xi, 1[$. This however allows us to give the following time transform of (20). For any $c>0,0 \leq t \leq$ $A_{1}$ we have

$$
\begin{aligned}
& \ln \frac{1}{1-Z_{t}^{\xi}}-\ln \frac{1}{1-Z_{\xi+c}^{\xi}} \\
& \quad=\int_{\xi+c}^{t} \frac{g_{s}}{\left(1-Z_{s}^{\xi}\right) \gamma\left(Y_{s}\right)} d \tilde{Y}_{s}-\int_{\xi+c}^{t} \frac{g_{s}}{1-Z_{s}^{\xi}} d B_{s}^{\xi}-\frac{1}{2} \int_{\xi+c}^{t}\left(\frac{g_{s}}{\left(1-Z_{s}^{\xi}\right) \gamma\left(Y_{s}\right)}\right)^{2} d s
\end{aligned}
$$

Formulas analogous to $(20)$ and $(22)$ for $\tau_{0}$ and $\xi_{0}$ with respect to $Z^{\tau_{0}}, Z^{\xi_{0}}$ hold. Now $\xi_{0} \leq \xi$ implies that for any $c>0$

$$
\ln \frac{1}{1-Z_{\xi_{0}+c}^{\xi_{0}}} \leq \ln \frac{1}{1-Z_{\xi+c}^{\xi}} \quad \text { at least on } \quad\left\{\xi_{0}=\xi\right\} .
$$

As a consequence of proposition 3.2 for any $0<t \leq 1$

$$
\int_{\xi_{0}}^{t}\left(\frac{g_{s}^{0}}{1-Z_{s}^{\xi_{0}}}\right)^{2} d s=\infty
$$

on a set of positive probability in $\left\{\xi_{0}=\xi\right\}$. By $\gamma^{2} \geq 1$ the same holds true for $\int_{\xi_{0}}^{1}\left(\frac{g_{s}^{0}}{\left(1-Z_{s}^{\xi_{0}}\right) \gamma\left(Y_{s}\right)}\right)^{2} d s$. Now by the relationship between the set of finite quadratic variation and martingale convergence (see Revuz, Yor [36], p.183) we must have

$$
\infty=\lim _{c \downarrow 0} \ln \frac{1}{1-Z_{\xi_{0}+c}^{\xi_{0}}} \leq \lim _{c \downarrow 0} \ln \frac{1}{1-Z_{\xi+c}^{\xi}}
$$

on a set of positive probability in $\left\{\xi_{0}=\xi\right\}$. By the same relationship (22) now entails

$$
\int_{\xi}^{t}\left(\frac{g_{s}}{\left(1-Z_{s}^{\xi}\right) \gamma\left(Y_{s}\right)}\right)^{2} d s=\infty
$$

for any $0<t \leq 1$ on a set of positive probability in $\left\{\xi_{0}=\xi\right\}$. Retransferring this to the original scale finally yields the desired statement

$$
\int_{\tau}^{t}\left(\frac{f_{s}}{1-Z_{s}^{\tau}}\right)^{2} d s=\infty
$$

for any $0<t \leq 1$ on a set of positive probability. We obtain the required degeneracy result.

Proposition 5.1 For any $t \in[0,1]$ we have

$$
\int_{0}^{t} \alpha_{s}^{2} d s=\infty
$$

on a set of positive measure. 


\section{Proof:}

On $] \tau, 1\left[, \alpha=\frac{f}{1-Z^{\tau}}\right.$. On a set of positive probability intersecting non-trivially with the one fixed above, $\tau<t$.

Our main result claims again that the time of the maximum of $X$ allows for arbitrage possibilities.

Theorem 5.1 Suppose the insider's filtration $\mathcal{G}$ is the progressive enlargement of $\mathcal{F}$ with $\tau$, the time at which the regular recurrent diffusion $Y$ satisfying the stochastic differential equation

$$
d Y_{t}=\gamma\left(Y_{t}\right) d W_{t}+c\left(Y_{t}\right) d t
$$

takes its maximum. The coefficients $\gamma$ and $c$ are such that $\gamma^{2}>\rho$ for some positive constant $\rho$ and $\frac{c^{2}}{\gamma^{2}}$ is locally integrable on $\mathbf{R}$. Then in the insider's model $R$ does not satisfy the condition (NFLVR).

\section{Proof:}

Combine Propositions 5.1 and 1.1.

\section{References}

[1] Amendinger, J. Initial Enlargement of Filtrations and Additional Information in Financial Markets. Thesis. TU Berlin, 1999.

[2] Amendinger, J., Imkeller, P., Schweizer, M. Additional logarithmic utility of an insider. Stoch. Proc. Appl. 75 (1998), 263-286.

[3] Amendinger, J., Becherer, D., Schweizer, M. Quantifying the value of initial investment information. Discussion paper 41/2000, Sonderforschungsbereich 373, Humboldt Univ., Berlin (2000).

[4] Back, K. Insider Trading in Continuous Time. Review of Financial Studies 5 (1992), 387-409.

[5] Back, K. Asymmetric Information and Options. Review of Financial Studies 6 (1993), 435-472.

[6] Cho, K.-H., El Karoui, N. Continuous Auction Equilibrium with Asymmetric Information: Non-linear Equilibria and Risk Aversion. Working paper 1998.

[7] Choulli, T., Krawczyk, L., Stricker, C. $\mathcal{E}$-martingales and their applications in mathematical finance. Annals of Probab. 26 (1998), 853-876.

[8] Choulli, T., Krawczyk, L., Stricker, C. On Fefferman and Burkholder-Davis-Gundy inequalities for $\mathcal{E}$-martingales. Probab. Theory Relat. Fields 113 (1999), 571-597. 
[9] Chaleyat-Maurel, M. , Jeulin, T. Grossissement Gaussien de la filtration Brownienne. in: Grossissements de filtrations: exemples et applications. T. Jeulin, M.Yor (eds.). LNM 1118. Springer: Berlin 1985.

[10] Delbaen, F., Schachermayer, W. A general version of the fundamental theorem of asset pricing. Math. Ann. 300 (1994), 463-520.

[11] Delbaen, F., Schachermayer, W. The existence of absolutly continuous local martingale measures. Ann. of Appl. Probab. 5 (1995), 926-945.

[12] Delbaen, F., Schachermayer, W. The variance-optimal martingale measure for continuous processes. Bernoulli. 2 (1996), 81-105.

[13] Delbaen, F., Schachermayer, W. Arbitrage possibilities in Bessel processes and their relations to local martingales. Probab. Theory Relat. Fields 102 (1995), 357366.

[14] Denis, L., Grorud, A., Pontier, M. Formes de Dirichlet sur un espace de WienerPoisson. Application au grossissement de filtrations. Working paper (1998).

[15] Duffie, D., Huang, C.-F. Multiperiod Security Markets with Differential Information. Journal of Mathematical Economics 15 (1986), 283-303.

[16] El Karoui, N., Quenez, M.C. Nonlinear pricing theory and backward stochastic differential equations. Financial Mathematics, LNM 1656 (1997), 191-246.

[17] Elliott, R. J., Geman, H., Korkie, B. M. Portfolio optimization and contingent claim pricing with different information. Stochastics and Stochastics Reports 60 (1997), 185-203.

[18] Föllmer, H., Imkeller, P. Anticipation cancelled by a Girsanov transformation: a paradox on Wiener space. Ann. Inst. H. Poincaré 29 (1993), 569-586.

[19] Grorud, A., Pontier, M. Insider Trading in a Continuous Time Market Model. International Journal of Theoretical and Applied Finance 1 (1998), 331-347.

[20] Grorud, A., Pontier, M. Comment détecter le délit d'initiés? Working paper 1999.

[21] Grorud, A., Pontier, M. Etude des probabilités neutres au risque dans le cadre d'asymétrie d'information sur un marché financier. Preprint, Univ. de Provence, Univ. de Toulouse.

[22] Imkeller, P. Enlargement of the Wiener filtration by an absolutely continuous random variable via Malliavin's calculus. Probab. Th. Rel. Fields 106 (1996), 105-135.

[23] Imkeller, P. Enlargement of the Wiener filtration by a manifold valued random element via Malliavin's calculus. Statistics and Control of Stochastic Processes. The Liptser Festschrift. Y. M. Kabanov, B. L. Rosovskii, A. N. Shiryaev, eds. World Scientific: Singapore 1997. 
[24] Imkeller, P., Pontier, M., Weisz, F. Free lunch and arbitrage possibilities in a financial market model with an insider. Stoch. Proc. Appl. 92 (2001), 103-130.

[25] Ito, K., Mc Kean, H. P. Diffusion processes and their sample paths. Springer: Berlin 1974.

[26] Jacod, J. Grossissement initial, hypothèse (H'), et théorème de Girsanov. in: Grossissements de filtrations: exemples et applications. T. Jeulin, M.Yor (eds.). LNM 1118. Springer: Berlin 1985.

[27] Jeanblanc, M., Rutkowski, M. Modelling of default risk: an overview. Preprint, Univ. Evry (1999).

[28] Jeulin, T. Semi-martingales et grossissement de filtration. LNM 833. Springer: Berlin 1980.

[29] Karatzas, I., Pikovsky, I. Anticipative Portfolio Optimization. Advances in Applied Probability 28 (1996), 1095-1122.

[30] Karatzas, I., Shreve, S. Brownian motion and stochastic calculus. Springer: Berlin 1988.

[31] Karatzas, I., Shreve, S. Methods of Mathematical Finance. Springer: Berlin 1998.

[32] Meyer, P.A. Sur un théorème de Jacod. Sém. de Probabilités XII. LNM 649. Springer: Berlin 1978.

[33] Kyle, A. Continuous Auctions and Insider Trading. Econometrica 53 (1985), 13151335 .

[34] O’Hara, M. Market Microstructure Theory. Blackwell 1995.

[35] Pikovsky, I.Market Equilibrium with Differential Information. Preprint, Columbia University (1999).

[36] Revuz, D., Yor, M. Continuous martingales and Brownian motion. (3 rd edition) Springer: Berlin 1999.

[37] Wu, C. T. Construction of Brownian Motions in Enlarged Filtrations and their Role in Mathematical Models of Insider Trading. Dissertation, HU Berlin 1999.

[38] Yor, M. Grossissement de filtrations et absolue continuité de noyaux. in: Grossissements de filtrations: exemples et applications. T. Jeulin, M.Yor (eds.). LNM 1118. Springer: Berlin 1985.

[39] Yor, M. Entropie d'une partition, et grossissement initial d'une filtration. in: Grossissements de filtrations: exemples et applications. T. Jeulin, M.Yor (eds.). LNM 1118. Springer: Berlin 1985. 
[40] Yor, M. Inégalités de martingales continues arrêtées à un temps quelconque, I: théorèmes généraux. in: Grossissements de filtrations: exemples et applications. T. Jeulin, M.Yor (eds.). LNM 1118. Springer: Berlin 1985.

[41] Yor, M. Inégalités de martingales continues arrêtées à un temps quelconque, II: le rôle de certains espaces BMO. in: Grossissements de filtrations: exemples et applications. T. Jeulin, M.Yor (eds.). LNM 1118. Springer: Berlin 1985.

[42] Yor, M. Some aspects of Brownian motion. Birkhäuser: Basel 1997. 